Research Journal of Biological Sciences 14 (2): 7-14, 2019

ISSN: $1815-8846$

(C) Medwell Journals, 2019

\title{
Detection of Tuberculosis Necrotizing Toxin Isolated from Mycobacterium tuberculosis Using Molecular Methods
}

\author{
Mujahid Khalaf Ali \\ Department of Microbiology, College of Medicine, University of Tikrit, Tikrit, Iraq
}

\begin{abstract}
To exhibit an incorporated atomic science devoted system for tuberculosis diagnosis. Mycobacterium tuberculosis (MTB) instigates putrefaction of infected cells to avoid immune reactions. As of late we found that MTB uses the protein CpnT to execute human macrophages by discharging its C-terminal area, named Tuberculosis Necrotizing Toxin (TNT) that incites putrefaction by an obscure component. The TNT controls the cytosol of MTB-infected macrophages where it hydrolyzes the main element co-catalyst Nicotinamide Adenine Dinucleotide $\left(\mathrm{NAD}^{+}\right)$. Articulation or infusion of a non-synergist TNT mutant demonstrated no cytotoxicity in macrophages or zebrafish zygotes, separately, exhibiting that the $\mathrm{NAD}^{+}$-glycohydrolase action is required for TNT-prompted cell demise. To anticipate self-harming, MTB produces a Immunity Factor for TNT (IFT) that ties TNT and represses its action. The precious stone structure of the TNT-IFT complex uncovered a novel $\mathrm{NAD}^{+}$-glycohydrolase overlap of TNT which constitutes the establishing individual from a toxin family across the board in pathogenic micro-organisms.
\end{abstract}

$\underline{\text { Key words: C-terminal, Mycobacterium tuberculosis, toxin, molecular, non-synergist, co-catalyst }}$

\section{INTRODUCTION}

Tuberculosis is a profoundly irresistible sickness and a worldwide wellbeing risk. Survival inside macrophages is a key component of Mycobacterium tuberculosis (MTB) pathogenesis and is essential to a determined disease in the human hostl. The fight amongst MTB and the human immune system to dominance the destiny of infected macrophages is basic in deciding the result of the contamination. The capacity to command the planning and method of host cell passing assumes a vital part in numerous microbial diseases.

Mycobacterium tuberculosis (MTB) is a pathogenic bacterial animal groups in the class Mycobacterium and the causative specialist of most instances of tuberculosis. According to Koch, $M$. tuberculosis has an unordinary, waxy covering on the cell surface (main elementlymycolic corrosive) which makes the cells impenetrable to gram recoloring; Corrosive quick systems are utilized which was in the year 1882. The physiology of M. tuberculosis is very vigorous and requires abnormal amounts of oxygen. Main elemently a pathogen of the mammalian respiratory system, MTB contaminates the lungs, causing tuberculosis.

M. tuberculosis expects oxygen to develop. It doesn't hold any basic bacteriological stain because of high lipid content in its divider and accordingly is neither Gram-positive nor Gram-negative, subsequently Ziehl-Neelsen recoloring or corrosive quick recoloring is utilized. While Mycobacteria don't appear to fit the Gram-positive classification from an experimental point of view (i.e., they don't hold the precious stone violet stain), they are delegated corrosive quick Gram-positive microorganisms because of their absence of an external cell film.

M. tuberculosis partitions each $15-20 \mathrm{~h}$ which is to a great degree ease back contrasted with other microscopic organisms which have a tendency to have division times estimated in minutes (Escherichia coli can separate generally at regular intervals). Its strange cell divider, wealthy in lipids (e.g., mycolic corrosive) is likely in charge of this opposition and is a key destructiveness factor. At the point when in the lungs, $M$. tuberculosis is taken up by alveolar macrophages, however, they can't process the bacterium. Its cell divider keeps the combination of the phagosome with a lysosome. In particular, $M$. tuberculosis hinders the spanning particle, Early Endosomal Autoantigen 1 (EEA1) in any case, this barricade does not avert combination of vesicles loaded up with supplements. Thus, the microorganisms duplicate unchecked inside the macrophage.

In $\mathrm{TB}$ congestion is common to the point that individuals are probably going to live in dull, unventilated rooms and in this manner they will probably be infected by TB and to get extensive measurements of the bacilli. Patient's protection from the malady is lessened, especially by lack of healthy sustenance and different ailments, for example, ignored tropical infections. 
Extraordinary neediness and every one of its backups ailing health, congestion or vagrancy, habit and absence of access to medicinal services are the real main thrusts basic the nearness and spread of $\mathrm{TB}$, incorporating the present increments in tuberculosis caused by various drug-safe and broadly drug safe straits.

What's more, the expansion in TB in late decades is specifically associated with the HIV plague and in nations with high HIV pervasiveness, the quantity of new TB cases has tripled in the previous 15 years. Creating nations endure the worst part of the scourges of AIDS and TB and the administration of patients with the two maladies represents a specific test in these settings. The reemergence of tuberculosis as a vital general medical problem and the spread of drug-safe tuberculosis have underlined the requirement for fast diagnosis. Notwithstanding, the standard culture strategies as of now being used are very moderate. Recognition of mycobacterial development on customary Lowenstein-Jensen medium expects 4 to about 2 months. Likewise, it requires specialized abilities and a high multifaceted nature bacteriology research center. The requirement for speed is additionally a factor when the consequences of tests have a positive effect to enable disease to command choices with respect to persistent separation and helpful administration (Alcaide et al., 2000; Badak et al., 1997; Behar et al., 2011; Divangahi et al., 2013; Hanna et al., 1999; Heep et al., 2000; Henkel et al., 2010).

It is in these kind of circumstances that sub-atomic demonstrative techniques can give the information required all the more quickly and much of the time it is more financially savvy than conventional culture strategies. Four years prior, we chose to build up a system of TB diagnosis that could satisfy these necessities. In this study we displayed a novel low many-sided quality/cost incorporated sub-atomic science committed system for the quick discovery of TB.

Destructive MTB initiates putrefaction of infected cells and smothers have cell apoptosis to avoid immune reactions and disperse. Executing of infected macrophages by corruption is reliant on the ESX-1 emission system and has been credited to the film action of ESAT-6. Interestingly, apoptosis of infected macrophages is instigated by the host immune system to put hold onthe bacterial disease. Along these lines, apoptotic bodies containing MTB are rummaged by other initiated macrophages or taken up by dendritic cells, to encourage the preparing of antigen-particular $\mathrm{T}$ cells to invigorate versatile invulnerability. In this manner the destiny of infected macrophages significantly influences have protection from MTB (Gordon et al., 2009;
Mukhopadhyay et al., 2012; Bartfai et al., 2001; Forrellad et al., 2013; Smith et al., 2008; Lee et al., 2011; Abdallah et al., 2011).

The atomic components by which MTB prompts macrophage rot are to a great extent obscure. Numerous bacterial pathogens use dangerous proteins to slaughter have cells. The absence of any protein in the MTB genome with homologs of known bacterial toxins and the inability to disengage discharged proteins with solid poisonous quality against have cells prompted the across the board conviction that MTB does not encode these established harmfulness factors. In any case, this worldview was tested by our revelation of the external film protein $\mathrm{CpnT}$ as the primary cytotoxicity factor of MTB in macrophages. CpnT is used by MTB to discharge its lethal C-terminal space (deposits 651-846) which is adequate to initiate necrotic demise in have cells by an obscure system. Subsequently, we named the emitted C-terminal space of CpnT as TNT (tuberculosis necrotizing toxin).

In this examination, we set out to distinguish the component of TNT-instigated necrotic cell passing at the utilitarian and basic level. We show that TNT has strong $\mathrm{NAD}^{+}$-glycohydrolase movement which drains cellular $\mathrm{NAD}^{+}$Pools bringing about host cell demise. TNT mutants with lower or revoked $\mathrm{NAD}^{+}$-glycohydrolase action, indicate decreased or no cytotoxicity, individually, in macrophages and in zebrafish building up a connection between the enzymatic action and lethality of TNT. We additionally distinguish an endogenous MTB protein which goes about as an antibody toxin for TNT to avoid self-harming. The precious stone structure of TNT in complex with its antibody toxin demonstrates a novel $\mathrm{NAD}^{+}$official and hydrolysis module unmistakable from that of known $\mathrm{NAD}^{+}$-using toxins.

\section{MATERIALS AND METHODS}

TNT hydrolyzes NAD ${ }^{+}$: Recombinant articulation of TNT (3' end of Rv3903c encoding the discharged C-terminal area) is harmful in all tried prokaryotic and eukaryotic cells, proposing a typical cellular target. Profound sequencing of $E$. coli RNA uncovered that TNT articulation actuated nadA and $\mathrm{B}$ translation by 16 -and 44-overlay, individually. These qualities encode enter compounds in Nicotinamide Adenine Dinucleotide $\left(\mathrm{NAD}^{+}\right)$biosynthesis recommending a connection amongst TNT and $\mathrm{NAD}^{+}$. Without a doubt, TNT articulation totally exhausted $\mathrm{NAD}^{+}$in $E$. coli and Jurkat T-cells, showing an association of TNT in corruption of cellular $\mathrm{NAD}^{+}$. Cleansed recombinant TNT hydrolyzed $\mathrm{NAD}^{+}$in vitro and was restrained by a TNT-particular immune response exhibiting that TNT corrupts $\mathrm{NAD}^{+}$. 

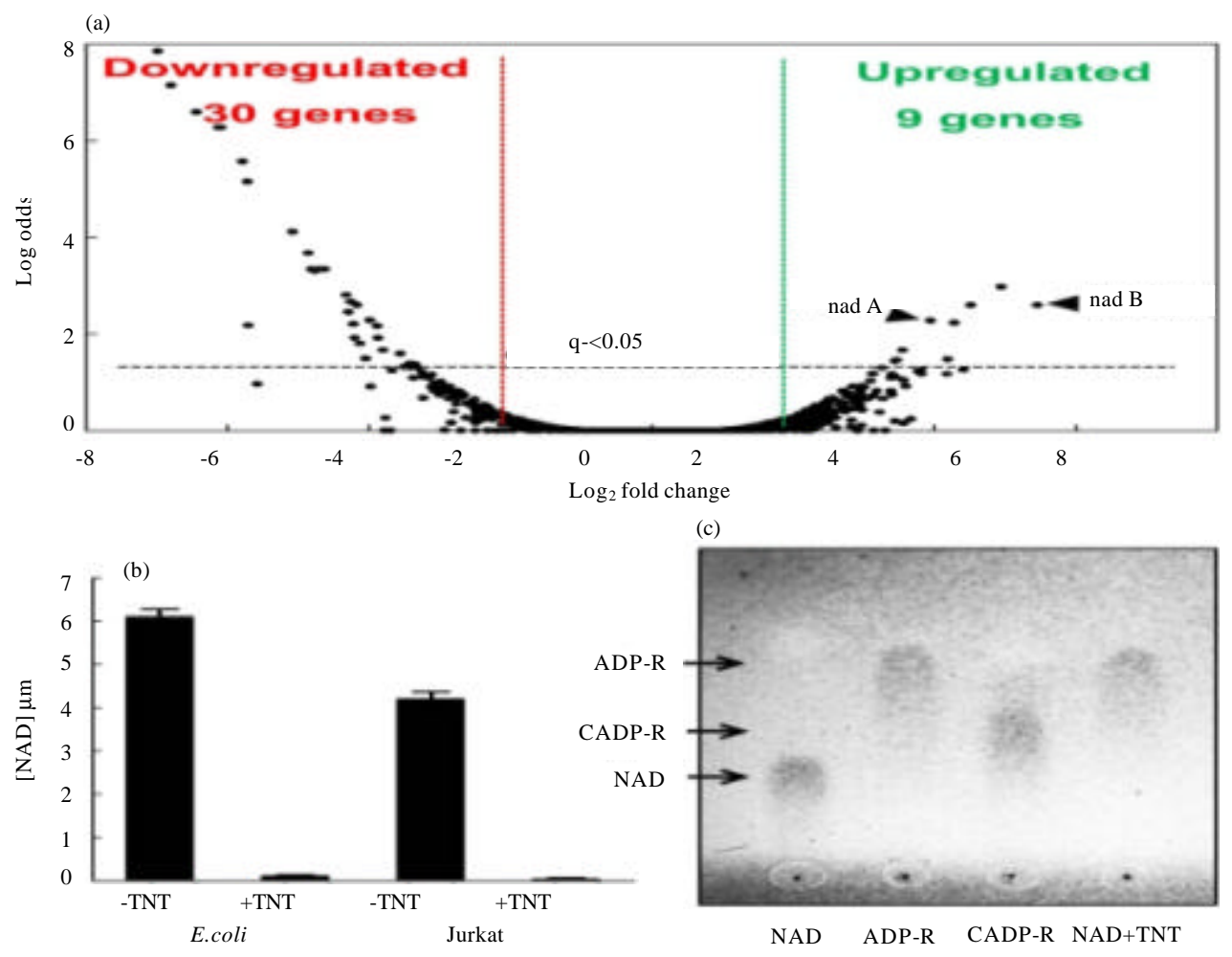

(c)

Fig. 1: a-c) TNT reduces cellular $\mathrm{NAD}^{+}$levels by degradation

$\mathrm{NAD}^{+}$is a main element coenzyme in numerous redox reactions and additionally a substrate for $\mathrm{NAD}^{+}$-expending catalysts that assume essential parts in transcriptional direction and life span. Consumption of cytosolic $\mathrm{NAD}^{+}$bargains ATP age by glycolysis and prompts necrotic cell death. Subsequently, $\mathrm{NAD}^{+}$ hydrolysis may clarify TNT's cytotoxicity. All known $\mathrm{NAD}^{+}$-degrading enzymes release nicotinamide and a second reaction product that varies with the specific hydrolytic mechanism. $\mathrm{NAD}^{+}$-glycohydrolases and ADP-ribosyl-cyclases create ADP-ribose and cyclic DP-ribose, individually while ADP-ribosyltransferases join ADP-ribose to a particular target protein.

Thin layer chromatography uncovered that TNT is a glycohydrolase that proficiently separates $\mathrm{NAD}^{+}$into nicotinamide and $\mathrm{ADP}$-ribose in vitro without some other protein. A dynamic examination of $\mathrm{NAD}^{+}$hydrolysis by TNT yielded a Michaelis consistent $\mathrm{km}$ of $614 \pm 43 \mu \mathrm{m}$, close to the physiological NAD ${ }^{+}$grouping of $\sim 500-600 \mu \mathrm{m}$ in human cells, a turnover number kcat of $52 \mathrm{~s}-1$ and a reactant effectiveness $\mathrm{kcat} / \mathrm{km}$ of $8.4 \times 104 \mathrm{~m}-1 \mathrm{~s}-1$. TNT has maximal action at $\mathrm{pH} 6.5$, yet is just possibly dynamic at $\mathrm{pH} 5.5$ recommending that TNT isn't dynamic in a develop phagolysosome. TNT is likewise strikingly warm stable holding roughly half of its action after delayed warming at $95^{\circ} \mathrm{C}$. The $\mathrm{pH}$ reliance and warmth security of TNT coordinate the uncharacterized $\mathrm{NAD}^{+}$-glycohydrolase action distinguished in MTB cell extricates 50 years back (Fig. 1).

The MTB protein Rv3902 $\mathrm{c}$ binds to and inactivates TNT: The main other known bacterial $\mathrm{NAD}^{+}$-glycohydrolase is the discharged toxin SPN of Streptococcus S. pyogenes. $S$. pyogenes ensures itself against the lethality of SPN by delivering the resistance factor IFS which is encoded in the SPN operon and firmly ties to and inactivates SPN. Nonetheless, SPN and IFS have no grouping likenesses to any MTB protein. Since, TNT articulation is dangerous in MTB (not appeared), we contemplated that Rv3902c, the last quality in the $\mathrm{CpnT}$ operon, may encode an antitoxin to TNT. To be sure, equimolar measures of decontaminated recombinant Rv3902c protein totally hindered TNT $\mathrm{NAD}^{+}$-glycohydrolase action. Surface Plasmon Reverberation (SPR) tests utilizing cleaned MBP-TNT and Rv3902c proteins uncovered that the two proteins frame a steady complex with a separation consistent $\mathrm{KD}$ of $2.3 \times 100-10 \mathrm{~m}$. Amazingly, an uncharacterized warm labile "inhibitor" of the MTB $\mathrm{NAD}^{+}$-glycohydrolase movement was additionally, portrayed in the 1960 's. In concurrence with this report, collapsing ponders uncovered that a complex of Rv3902c with TNT denatured irreversibly with a clear softening 
temperature of $\sim 65.4^{\circ} \mathrm{C}$, prompting sensational conformational changes of the TNT-IFT complex and freeing TNT taken together, these tests show that Rv3902c restrains the $\mathrm{NAD}^{+}$-glycohydrolase action of TNT by complex development and constitutes the main inhibitor of a discharged toxin in MTB. Consequently we named Rv3902c as Immunity Factor for TNT (IFT).

\section{RESULTS AND DISCUSSION}

The atomic structure of the TNT-IFT complex: The revelation of IFT empowered us to filter milligram amounts of the TNT-IFT complex from $E$. coli after co-articulation of the relating qualities. We solidified the TNT-IFT complex at physiological $\mathrm{pH}$ and decided its structure to a Rwork/Rfree of $12.8 / 14.3 \%$, at $1.1 \AA$ goals. The TNT-IFT complex receives a blocky compliance of $50 \times 50 \times 35 \AA 3$ that takes after a 'ball-in-a-hand'. IFT ('ball') fills a profound depression in TNT ('hand') that covers $\sim 20 \%$ of the perplexing dissolvable open zone $(\sim 3,490 \AA 2)$. Steady with the picomolar $\mathrm{KD}$ estimated by SPR, the broad restricting interface amongst TNT and IFT includes roughly $33 \%$ of the buildups in every protein and results in more than 50, principally electrostatic, contacts. Strikingly, both TNT and IFT have negligible interior hydrophobic centers and as much as $97 \%$ of their deposits are uncovered on the structure surface, bringing about gigantic anticipated solvation energies $(\Delta \mathrm{Gf}=-157$ and $163 \mathrm{kcal} / \mathrm{mol}$ for TNT and IFT, separately).

The TNT structure comprises of two locales: a 'thumb' (buildups 648-736) and a'palm-space' (deposits $747-846$ ). The $75 \%$ of the buildups in the thumb space receive an arbitrary wound compliance hindered by two shorts $\beta$-helices $(\beta 1, \beta 2)$ and two antiparallel $\beta$-strands $(\beta 3, \beta 4)$. The compliances of both main element and side chains in the thumb space between two gem types of the TNT-IFT complex uncovered unobtrusive basic contrasts between deposits 669-679, conceivably caused by the absence of precious stone contacts in this district and underscoring the basic pliancy of this area. The thumb folds over the palm space which is shaped by a six-stranded $\beta$-sheet encompassed by two short $\beta$-helices and two $3 / 10$ helices.

Four strands, orchestrated into two about symmetrical antiparallel $\beta$-hairpins $(\beta 12-\beta 13$ and $\beta 7-\beta 11)$ are significantly more and take after the four fingers of a hand. IFT receives a globular structure that comprises of a $\beta$-rich space containing two antiparallel $\beta$-sheets ( $\beta 1-\beta 5$ and $\beta 7-\beta 9$ ) and a helical area, uncovered on the contrary surface, shaped by $4 \beta$-helices. Between the TNT palm and the thumb area is a $\sim 15 \AA$ profound cleft possessed by two IFT $\beta$-turns named TNT-collaboration circles 1 and
Table 1: Crystallographic information gathering and refinement insights

\begin{tabular}{|c|c|c|}
\hline Variables & $\begin{array}{l}\text { TNT-IFT } \\
\text { crystal form } 1\end{array}$ & $\begin{array}{l}\text { TNT-IFT } \\
\text { crystal form } 2\end{array}$ \\
\hline \multicolumn{3}{|l|}{ Data collection } \\
\hline Space group & $\mathrm{P} 2{ }_{1} 2_{1} 2_{1}$ & $\mathrm{P} 22_{1} 2_{1} 2_{1}$ \\
\hline \multicolumn{3}{|l|}{ Cell dimensions } \\
\hline $\mathrm{a}, \mathrm{b}, \mathrm{c}(\AA)$ & $70.6,86.2,62.6$ & $75.3,85.3,63.2$ \\
\hline$\alpha, \beta, \gamma\left({ }^{\circ}\right)$ & $90.0,90.0,90.0$ & $90.0,90.0,90.0$ \\
\hline Wavelength $(\AA)$ & 0.97 & 0.97 \\
\hline Resolution $(\AA)$ & $20-1.10(1.14-1.10)$ & $20-1.9(1.97-1.90)$ \\
\hline $\mathrm{R}_{\mathrm{sym}}$ & $5.7(50.2)$ & $7.2(33.5)$ \\
\hline $\mathrm{I} / \sigma \mathrm{I}$ & $29.5(4.5)$ & $66.6(10.8)$ \\
\hline Completeness (\%) & $90.8(55.5)$ & $99.5(93.3)$ \\
\hline Redundancy & $12.6(8.8)$ & $13.2(9.7)$ \\
\hline \multicolumn{3}{|l|}{ Refinement } \\
\hline Resolution $(\AA)$ & $1.0-1.1$ & $20-1.9$ \\
\hline No. reflections & 133,725 & 32,610 \\
\hline $\mathrm{R}_{\text {work }} / \mathrm{R}_{\text {free }}$ & $12.8 / 14.3$ & $17.0 / 20.8$ \\
\hline \multicolumn{3}{|l|}{ No. atoms } \\
\hline Protein & 2,990 & 2,889 \\
\hline Water & 506 & 461 \\
\hline \multicolumn{3}{|l|}{ B-factors $\left(\AA^{2}\right)$} \\
\hline Protein (TNT/IFT) & $13.6 / 15.2$ & $25.5 / 26.4$ \\
\hline Water & 29.9 & 36.1 \\
\hline \multicolumn{3}{|l|}{ Rms deviations } \\
\hline Bond lengths $(\AA)$ & 0.006 & 0.008 \\
\hline Bond angles $\left({ }^{\circ}\right)$ & 1.09 & 1.19 \\
\hline
\end{tabular}

2 that enter profoundly inside the TNT center and make broad electrostatic contacts. Despite the fact that the structure of IFT in complex with TNT superimposes well to the structure of free IFT (rmsd $\sim 0.52 \AA$ ), the side chain adaptations in the TNT-collaboration circles (N36, K37, E40 and D58, R59, M60, S61) anticipating inside the TNT hole are extraordinary Table 1. Qualities in brackets are for most noteworthy goals shells.

Recognizable proof of putative $\mathrm{NAD}^{+}$-restricting site of TNT, TNT has no succession closeness to any protein of known capacity and a comprehensive look for comparable structures neglected to yield critical hits. In any case, a restriction based basic arrangement of TNT with the SPN glycohydrolase space and the ribosyltransferase areas of the diphtheria and cholera toxins uncovered a comparative essential design of the $\mathrm{NAD}^{+}$-restricting center. Recognizable contrasts are that the TNT center contains just six $\beta$-strands rather than seven found in all known $\mathrm{NAD}^{+}$-using toxins and is main elemently littler with just two short $\beta$-helices and two $3 / 10$ helices. In silico docking promptly put $\mathrm{NAD}^{+}$in the profound hole of TNT with an anticipated free-vitality of official of-8.7 $\mathrm{kcal} / \mathrm{mol}$. All arrangements share a comparable position of the nicotinamide amass that supplements profoundly inside the TNT hole. Investigation of the amino acids encompassing the putative $\mathrm{NAD}^{+}$-restricting pocket distinguished Q822, Y765 and R757 as buildups perhaps associated with $\mathrm{NAD}^{+}$-official and hydrolysis in light of comparative places of synergist amino acids of ADP-ribosylating toxins. Out of these buildups, the glutamine 822 is 
Res. J. Biol. Sci., 14 (2): 7-14, 2019

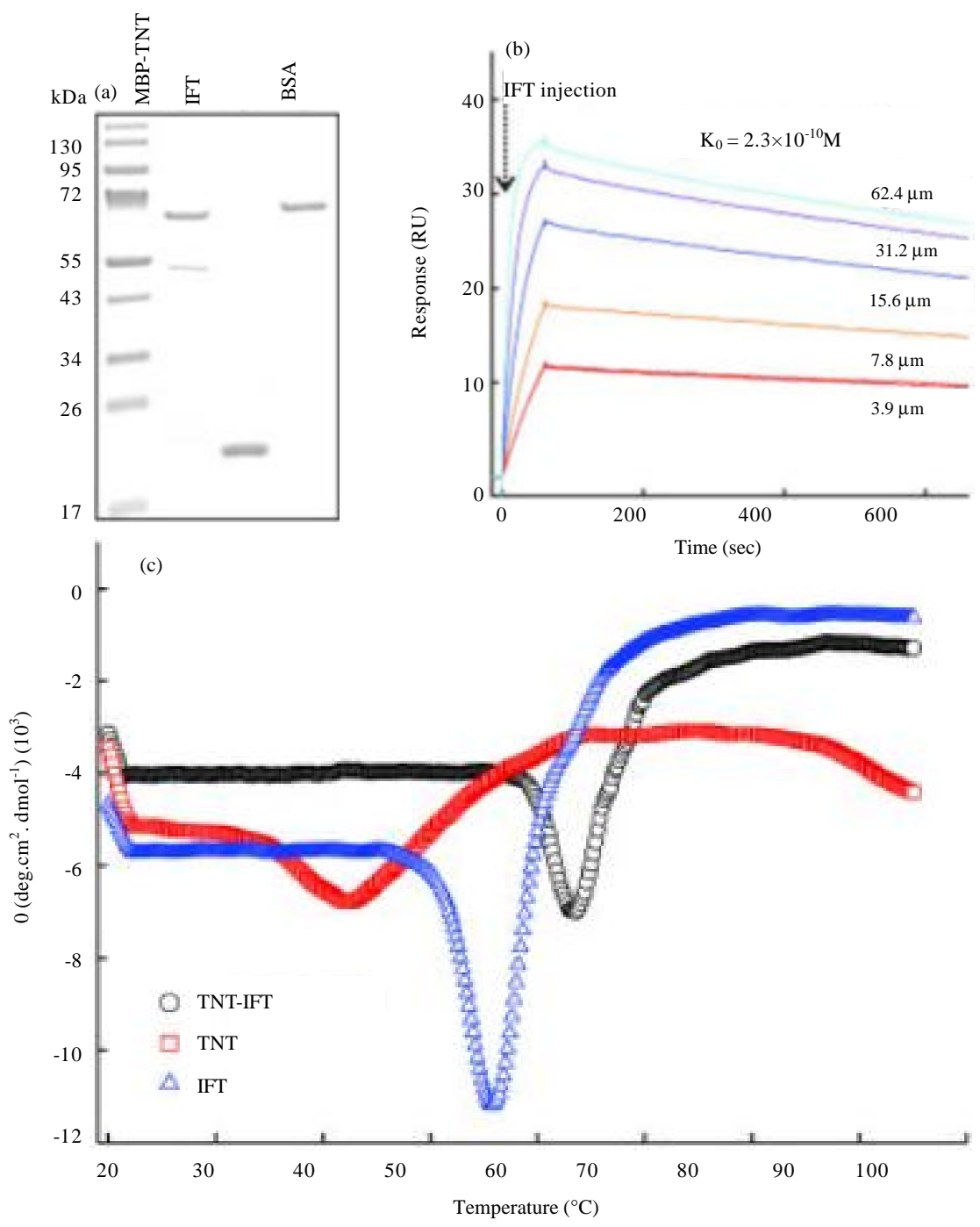

Fig. 2: a-c) M. tuberculosis delivers the endogenous invulnerability factor IFT that ties to and inactivates TNT

profoundly saved among TNT homologs. To decide the part of $\mathrm{Q} 822$ in $\mathrm{NAD}^{+}$hydrolysis, we created the TNTQ822A protein in E. coli within the sight of the antibody toxin IFT to avoid auxiliary transformations and isolated the complex by particular warm denaturation. Cleaned TNTQ822A kept up auxiliary honesty as dictated by IFT official, yet its $\mathrm{NAD}^{+}$-glycohydrolase action was diminished by two-overlay contrasted with wild-type TNT, proposing that glutamine 822 is vital, however, not main element, for authoritative and additionally, hydrolysis of $\mathrm{NAD}^{+}$. So, also the TNTY765A mutant protein additionally had diminished $\mathrm{NAD}^{+}$-glycohydrolase movement in vitro and cytotoxicity in macrophages. By differentiate, transformation of the comparing glutamine 148 and tyrosine 65 deposits in diphtheria toxin brought about the extraordinary loss of its ADP-ribosyltransferase and $\mathrm{NAD}^{+}$-glycohydrolase exercises, recommending that TNT utilizes an alternate instrument of $\mathrm{NAD}^{+}$authoritative and perhaps hydrolysis Fig. 2.

The exceptionally rationed G818 buildup likewise lines the putative $\mathrm{NAD}^{+}$restricting pocket of TNT. Be that as it may, the cleaned TNTG818V protein was unfurled as demonstrated by round about dichroism and absence of IFT authoritative (not appeared) and henceforth did not hydrolyze $\mathrm{NAD}^{+}$. Since, a few single mutants including TNTQ822A did not totally wipe out the $\mathrm{NAD}^{+}$-glycohydrolase movement of TNT, aside from the unfurled TNTQ818V mutant, we misused the incidental choice of an extra transformation, $\mathrm{H} 792 \mathrm{~N}$ in $E$. coli conveying a TNTQ822K articulation vector without IFT. For sure, the purged TNTH792N Q822K protein had no noticeable $\mathrm{NAD}^{+}$-glycohydrolase action in a 
delicate test with a discovery farthest point of $0.1 \mu \mathrm{m}$ $\mathrm{NAD}^{+}$, yet effectively bound to IFT in pull-down trials showing that the loss of $\mathrm{NAD}^{+}$-glycohydrolase action was not caused by hindered protein collapsing. Taken together, this mutational examination distinguishes the putative $\mathrm{NAD}^{+}$restricting site of TNT, yet in addition uncovers considerable contrasts contrasted with SPN and ADP-ribosylating toxins.

TNT interceded $\mathrm{NAD}^{+}$depletion causes macrophage cell termination: To evaluate whether $\mathrm{NAD}^{+}$depletion by TNT prompts cells termination, RAW 264.7 macrophages were fleetingly transfected with plasmids communicating egfp combinations with wt. TNT or mutant TNT genes. From that point, macrophage practicality was evaluated by $7 \mathrm{AAD}$ recoloring and stream cytometry. While wt. TNT executed over half of the transfected macrophages, the non-reactant TNTH792N Q822K mutant totally lost danger as did the unfurled TNT G818V mutant showing that macrophage cell passing is undoubtedly subject to the $\mathrm{NAD}^{+}$-glycohydrolase movement of TNT. The TNTQ822A mutant indicated middle cytotoxicity which is predictable with its halfway enzymatic movement. Articulation, of all TNT mutants was significantly higher than wtTNT as appeared by fluorescence of the GFP combination proteins, therefore, showing that the lower harmfulness was not because of lower articulation levels. To look at the part of the $\mathrm{NAD}^{+}$-glycohydrolase movement of TNT with regards to MTB disease, we contaminated THP-1 macrophages with wtMTB, the $\Delta \mathrm{CpnT}$ mutant and the $\Delta \mathrm{CpnT}$ mutant supplemented with CpnT encoding the wt. TNT or the non-reactant TNTH792N Q822K space. Macrophages contaminated with wt. MTB demonstrated an uncommon decrease in $\mathrm{NAD}^{+}$levels $24 \mathrm{~h}$ present disease interestingly on macrophages tainted with the MTB $\Delta \mathrm{CpnT}$ erasure mutant where $\mathrm{NAD}^{+}$levels stayed high. Vitally, the non-synergist TNTH792N Q822K mutant was communicated to comparative protein levels as wt TNT and demonstrated a comparative subcellular confinement design, yet did not diminish $\mathrm{NAD}^{+}$levels contrasted with that of the MTB $\Delta \mathrm{CpnT}$ erasure mutant. This outcome is predictable with the entire loss of $\mathrm{NAD}^{+}$-glycohydrolase movement of the TNTH792N Q822K mutant and exhibits that $\mathrm{NAD}^{+}$-depletion in macrophages contaminated with MTB for the most part relies upon the enzymatic action of TNT. It ought to be noticed that $\mathrm{NAD}^{+}$levels are additionally decreased in MTB-tainted macrophages by $40 \%$ through TNT-autonomous systems contrasted with uninfected macrophages.

To correspond the watched TNT-subordinate $\mathrm{NAD}^{+}$ depletion amid disease with the known cytotoxicity of MTB7, we gauged the suitability of macrophages tainted with MTB strains emitting wt. TNT or the chemically idle TNTH792N Q822K. Critically, the cytotoxicity of MTB discharging the non-reactant TNTH792N Q822K was lessened to indistinguishable level from that of the MTB CpnT erasure mutant. The elucidation of the contamination tests is confused by the constriction of the MTB CpnT erasure mutant in macrophages in light of the fact that the decreased bacillary load may likewise lessen cytotoxicity by instruments other than $\mathrm{NAD}^{+}$hydrolysis. In any case, the bacterial heap of the MTB strain communicating just the $\mathrm{N}$-terminal area of $\mathrm{CpnT}$ was expanded by 12-overlap, however, demonstrated indistinguishable cytotoxicity from the $\mathrm{CpnT}$ mutant showing that the watched contrast in cytotoxicity is likely not a result of less microscopic organisms but rather is reliant on TNT movement.

Besides, these outcomes connect $\mathrm{NAD}^{+}$levels to cytotoxicity and are additionally reliable with our perceptions that no $\mathrm{NAD}^{+}$is noticeable in Jurkat T cells 24 $\mathrm{h}$ after acceptance of TNT articulation and that over $90 \%$ of these phones kicked the bucket amid this time. Supplementation of this Jurkat $\mathrm{T}$ cell line with nicotinamide or nicotinic corrosive, both $\mathrm{NAD}^{+}$ antecedents that lift intracellular $\mathrm{NAD}^{+}$levels, lessened TNT-instigated lethality. This impact isn't because of hindrance of the enzymatic movement of TNT caused by expansion of nicotinamide or nicotinic corrosive. These $\mathrm{NAD}^{+}$forerunner supplementation tests bolster the immediate part of $\mathrm{NAD}^{+}$hydrolysis in the harmfulness of TNT. Taken together, these tests give convincing proof that $\mathrm{NAD}^{+}$hydrolysis by TNT is the main element harmfulness system of MTB in macrophages Fig. 3.

$\mathrm{NAD}^{+}$content in macrophages infected with MTB. THP-1 monocytes were separated with $100 \mathrm{ng} / \mathrm{mL}$ PMA for $24 \mathrm{~h}$ and in this manner infected with MTB endure a MOI of 20:1.24 h post contamination, macrophages were lysed in $0.025 \% \mathrm{SDS}$ to discharge all $\mathrm{NAD}^{+}$from the macrophages, however, not mycobacteria. The enzyfluo NAD/NADH unit was then used to measure the measure of $\mathrm{NAD}^{+}$in each example.

Intracellular recoloring: MTB infected RAW 264.7 macrophages on glass coverslips were settled with $4 \%$ paraformaldehyde for $1 \mathrm{~h}$ at room temperature and hence washed different occasions with PBS before recoloring. Cells were specifically permeabilized as depicted. In short, cells were permeabilized with $25 \mu \mathrm{g} / \mathrm{mL}$ digitonin in PBS alone for 5 min or in mix with $0.2 \%$ Triton-X100 in PBS for an extra $5 \mathrm{~min}$. At that point, cells were washed and hindered with PBS containing 1\% BSA (S-Buffer) for 20 min. From that point, polyclonal $\alpha$-TNT counter acting agent $(1: 75)$ or monoclonal $\alpha$-Ag85 immunizer $(1: 50)$ was utilized in S-Buffer and brooded with the cells for $60 \mathrm{~min}$. 
(a)

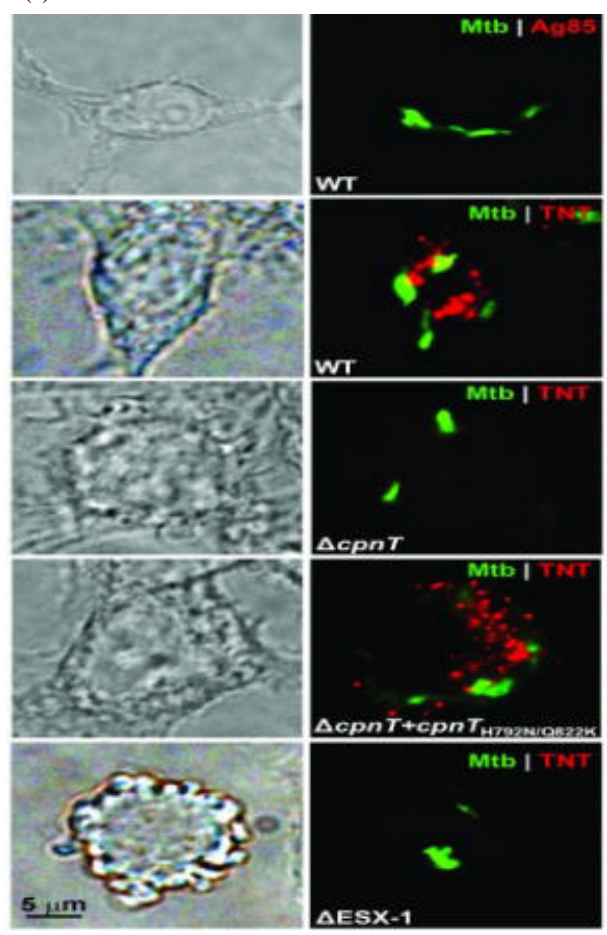

(b)

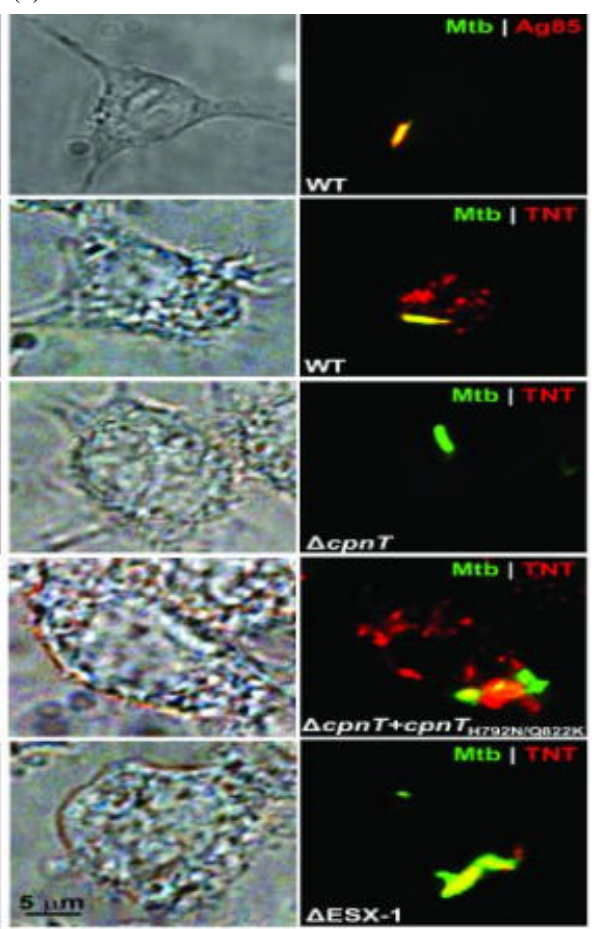

Fig. 3: $M$. tuberculosis secretes TNT into the Cytosol+Phagosome/(Digitonin+Triton $\times-100$ )

After 3 washes with PBS, auxiliary enemy of rabbit or hostile to mouse Alexa Fluor 555 (invitrogen) was utilized at a weakening of 1:1000 and hatched with the cells for 30 min. Cells were then washed 3 times with PBS and coverslips were mounted onto microscope slides with Fluor Save (Calbiochem) for fluorescence imaging. Imaging was performed utilizing an Axiovert 200 microscope (Carl Zeiss) outfitted with a $100 \times / 1.4$ Plan-Apochromat (Carl Zeiss). Pictures were recorded utilizing an AxioCamMRc camera (Zeiss) coupled to Axiovision V4.5 programming (Carl Zeiss).

TNT danger in Jurkat cells and protect by nicotinamide: Jurkat cells communicating TNT under an inducible system were pre-treated with either $5 \mathrm{~m}$ Mnicotinamide, 10 iM nicotinic corrosive or in mix for $2 \mathrm{~h}$. Now, articulation of TNT was prompted by expansion of $100 \mu \mathrm{g} / \mathrm{mL}$ doxycycline for $2 \mathrm{~h}$. Cells were therefore, washed free of doxycycline and hatched in general media for $24 \mathrm{~h}$. Cell feasibility was then surveyed utilizing the 7AAD color taken after by stream cytometry.

\section{CONCLUSION}

In this investigation, we set out to distinguish the component of TNT-instigated necrotic cell death at the practical and auxiliary level. We show that TNT has hearty $\mathrm{NAD}^{+}$-glycohydrolase movement which drains cellular $\mathrm{NAD}^{+}$pools bringing about host cell death. TNT mutants with lower or annulled $\mathrm{NAD}^{+}$-glycohydrolase action, demonstrate lessened or no cytotoxicity, individually in macrophages and in zebrafish building up a connection between the enzymatic movement and poisonous quality of TNT. We additionally, recognize an endogenous MTB protein which goes about as an immunizing agent toxin for TNT to avoid self-harming. The gem structure of TNT in complex with its neutralizing agent toxin demonstrates a novel $\mathrm{NAD}^{+}$authoritative and hydrolysis module unmistakable from that of known $\mathrm{NAD}^{+}$-using toxins.

Taking everything into account, the TB AccuProbe, the LiPA measure or rpoB sequencing are solid strategies that can be utilized in conjunction with the MGIT 960 system for clinical and reference labs to distinguish and recognize the $\mathrm{MTBC}$ with a turnaround time of under about 14 days. The tests (particularly the LiPA test) can likewise be useful in the distinguishing proof of the MTBC in a blended culture which may take a long time by ordinary techniques. The LiPA examine and rpoB sequencing likewise offer the ability of identifying RIF opposition in about 14 days for all separates or in 15-20 days in spread negative cases, together with the 
recognizable proof of the pathogen. The way that none of the sub-atomic techniques could distinguish the MTBC in every blended culture demonstrates that, at display, the immunization of both fluid and strong media and the affirmation of the sub-atomic outcomes by regular tests are still justified.

\section{REFERENCES}

Abdallah, A.M., J. Bestebroer, N.D. Savage, K. De Punder and M. Van Zon et al., 2011. Mycobacterial secretion systems ESX-1 and ESX-5 play distinct roles in host cell death and inflammasome activation. J. Immunol., 187: 4744-4753.

Alcaide, F., M.A. Benitez, J.M. Escriba and R. Martin, 2000. Evaluation of the BACTEC MGIT 960 and the $\mathrm{MB} / \mathrm{BacT}$ systems for recovery of mycobacteria from clinical specimens and for species identification by DNA AccuProbe. J. Clin. Microbiol., 38: 398-401.

Badak, F.Z., D.L. Kiska, M. O'Connell, C.M. Nycz and C. Hartley et al., 1997. Confirmation of the presence of Mycobacterium tuberculosis and other mycobacteria in Mycobacterial Growth Indicator Tubes (MGIT) by multiplex strand displacement amplification. J. Clin. Microbiol., 35: 1239-1243.

Bartfai, Z., A. Somoskovi, C. Kodmon, N. Szabo and E. Puskas et al., 2001. Molecular characterization of rifampin-resistant isolates of Mycobacterium tuberculosis from Hungary by DNA sequencing and the line probe assay. J. Clin. Microbiol., 39: 3736-3739.

Behar, S.M., C.J. Martin, M.G. Booty, T. Nishimura and X. Zhao et al., 2011. Apoptosis is an innate defense function of macrophages against Mycobacterium tuberculosis. Mucosal Immunol., 4: 279-287.
Divangahi, M., S.M. Behar and H. Remold, 2013. Dying to live: How the death modality of the infected macrophage affects immunity to tuberculosis. Adv. Exp. Med. Biol., 783: 103-120.

Forrellad, M.A., L.I. Klepp, A. Gioffre, Y. Sabio and J. Garcia et al., 2013. Virulence factors of the Mycobacterium tuberculosis complex. Virulence, 4 : 3-66.

Gordon, S.V., D. Bottai, R. Simeone, T.P. Stinear and R. Brosch, 2009. Pathogenicity in the tubercle bacillus: Molecular and evolutionary determinants. Bioessays, 31: 378-388.

Hanna, B.A., A. Ebrahimzadeh, L.B. Elliott, M.A. Morgan and S.M. Novak et al., 1999. Multicenter evaluation of the BACTEC MGIT 960 system for recovery of Mycobacteria. J. Clin. Microbiol., 37: 748-752.

Heep, M., U. Rieger, D. Beck and N. Lehn, 2000. Mutations in the beginning of the rpoBGene can induce resistance to Rifamycins in both helicobacter pylori and Mycobacterium tuberculosis. Antimicrob. Agents Chemother., 44: 1075-1077.

Henkel, J.S., M.R. Baldwin and J.T. Barbieri, 2010. Toxins from bacteria. EXS. Mol. Clin. Environ. Toxicol., 100: $1-29$.

Lee, J., T. Repasy, K. Papavinasasundaram, C. Sassetti and H. Kornfeld, 2011. Mycobacterium tuberculosis induces an atypical cell death mode to escape from infected macrophages. PloS one, 6: 1-13.

Mukhopadhyay, S., S. Nair and S. Ghosh, 2012. Pathogenesis in tuberculosis: Transcriptomic approaches to unraveling virulence mechanisms and finding new drug targets. FEMS Microbiol. Rev., 36: 463-485.

Smith, J., J. Manoranjan, M. Pan, A. Bohsali and J. Xu et al., 2008. Evidence for pore formation in host cell membranes by ESX-1-secreted ESAT-6 and its role in Mycobacterium marinum escape from the vacuole. Infect. Immun., 76: 5478-5487. 